\title{
Evaluating targets and costs of treatment for secondary hyperparathyroidism in incident dialysis patients: the FARO-2 study
}

This article was published in the following Dove Press journal: International Journal of Nephrology and Renovascular Disease 16 December 2014

Number of times this article has been viewed

\author{
Daniela Paola Roggeri' \\ Mario Cozzolino² \\ Sandro Mazzaferro ${ }^{3}$ \\ Diego Brancaccio ${ }^{4}$ \\ Ernesto Paoletti ${ }^{5}$ \\ Alessandro Roggeri' \\ Anna Maria Costanzo 6 \\ Umberto di Luzio \\ Paparatti ${ }^{6}$ \\ Vincenzo Festa ${ }^{6}$ \\ Piergiorgio Messa ${ }^{7}$ \\ 'ProCure Solutions, Nembro, \\ Bergamo, ${ }^{2}$ Department of Health \\ Sciences, University of Milan, Milan, \\ ${ }^{3}$ Department of Cardiovascular, \\ Respiratory, Nephrologic and \\ Geriatric Sciences, Sapienza \\ University of Rome, Rome, ${ }^{4}$ Dialysis \\ Unit NephroCare Simone Martini, \\ Milan, ${ }^{5}$ Department of Nephrology, \\ San Martino Hospital, Genoa, \\ ${ }^{6}$ AbbVie Italy, Campoverde, Latina, \\ ${ }^{7} \mathrm{Nephrology}$, Dialysis and Renal \\ Transplant, Fondazione Ca Granda \\ IRCCS Policlinico, Milan, Italy
}

On behalf of the FARO Study Group

Correspondence: Alessandro Roggeri ProCure Solutions, Via Camozzi I/C, 24027 Nembro, Italy

Tel +39035 52 I I 2 I

Email alessandro.roggeri@

procuresolutions.it
Background: The aim of this analysis was to estimate biochemical parameters and the costs of treatment of secondary hyperparathyroidism (SHPT) in a subpopulation of the FARO-2 study.

Methods: The FARO-2 observational study aimed at evaluating the patterns of treatment for SHPT in naïve hemodialysis patients. Data related to pharmacological treatments and biochemical parameters (parathyroid hormone [PTH], calcium, phosphate) were recorded at entry to hemodialysis (baseline) and 6 months later (second survey). The analysis was performed from the Italian National Health Service perspective.

Results: Two prominent treatment groups were identified, ie, one on oral calcitriol $(n=105)$ and the other on intravenous paricalcitol $(n=33)$; the intravenous calcitriol and intravenous paricalcitol + cinacalcet combination groups were not analyzed due to low patient numbers. At baseline, serum PTH levels were significantly higher in the intravenous paricalcitol group $(P<0.0001)$. At the second survey, the intravenous paricalcitol group showed a higher percentage of patients at target for PTH than in the oral calcitriol group without changing the percentage of patients at target for phosphate. Moreover, between baseline and the second survey, intravenous paricalcitol significantly increased both the percentage of patients at target for PTH $(P=0.033)$ and the percentage of patients at target for the combined endpoint $\mathrm{PTH}$, calcium, and phosphate $(P=0.001)$. The per-patient weekly pharmaceutical costs related to SHPT treatment, erythropoietin-stimulating agents and phosphate binders accounted for $186.32 €$ and $219.94 €$ at baseline for oral calcitriol and intravenous paricalcitol, respectively, while after 6 months, the costs were $180.51 €$ and $198.79 €$, respectively. Either at the beginning of dialysis or 6 months later, the total cost of SHPT treatment was not significantly lower in the oral calcitriol group compared with the intravenous paricalcitol group, with a difference among groups that decreased by $46 \%$ between the two observations. The cost of erythropoietin stimulating agents at the second survey was lower $(-22 \%)$ in the intravenous paricalcitol group than in the oral calcitriol group (132.13€ versus $168.36 €$, respectively).

Conclusion: Intravenous paricalcitol significantly increased the percentage of patients at target for the combined endpoint of PTH, calcium, and phosphate $(P=0.001)$. The total cost of treatment for the patients treated with intravenous paricalcitol 6 months after entry to dialysis was not significantly higher than the cost for patients treated with oral calcitriol.

Keywords: cost consequences analysis, therapeutic costs, outcomes, SHPT treatments, secondary hyperparathyroidism

\section{Introduction}

Chronic kidney disease (CKD) in all its stages is a relevant public health problem, recognized in 2011 by the World Health Organization as one of the chronic noncommunicable submit your manuscript | www.dovepress.com

Dovepress

http://dx.doi.org//0.2147/IJNRD.S7201।
International Journal of Nephrology and Renovascular Disease 20 I5:8 I-6

(c) (i) (5) 2015 Roggeri et al. This work is published by Dove Medical Press Limited, and licensed under Creative Commons Attribution - Non Commercial (unported, v3.0) License. The full terms of the License are available at http://creativecommons.org/licenses/by-nc/3.0/. Non-commercial uses of the work are permitted without any further permision how to request permission may be found at: http://www.dovepress.com/permissions.php 
diseases to fight in the coming decades and identified by the Centers for Disease Control and Prevention as a major medical priority in recent years. ${ }^{1}$

CKD is not only a clinical concern, but also a growing economic and organizational problem, as renal replacement therapy consumes a considerable proportion of health care resources. This problem in Italy is virtually unknown to the public and is still little known and largely underestimated by physicians and governing health authorities. The Italian Registry of Dialysis and Transplantation reported that the prevalence of patients on hemodialysis was 788 per million population, while the incidence was 162 per million population, corresponding to 42,488 patients on hemodialysis, 8,638 of whom are incident patients. ${ }^{2}$

CKD often affects elderly patients and is associated with important comorbidities (cardiac, vascular, osteoarticular, neuromuscular), with a relevant impact on patients' quality of life; $;^{3-6}$ a recent Italian study revealed that dialysis patients perceived an impaired quality of life, and hemodialysis patients in particular noticed a worse quality of life compared with peritoneal dialysis patients. ${ }^{?}$

From the economic point of view, in the USA, total Medicare expenditures for end-stage renal disease (ESRD) reached $\$ 33$ billion in 2010, accounting for more than $6 \%$ of the total Medicare budget. About $1.3 \%$ of Medicare patients had ESRD in 2010, yet accounted for 7.5\% of Medicare spending. Medicare costs for ESRD were $\$ 75,000$ per patient per year. ${ }^{8}$

Worldwide, several studies have reported relevant costs in the charge to health services associated with the treatment of ESRD patients; ${ }^{9-14}$ in Italy, these average yearly costs per hemodialysis patient were reported to range from about $36,000 €$ to $50,000 €$ (depending on the type of resources considered in the studies).

Secondary hyperparathyroidism (SHPT) is a frequent comorbidity in CKD and ESRD patients, and leads to bone and mineral metabolism disorders. Bone diseases represent only one of the consequences of SHPT, whereas the most dreaded are cardiovascular diseases and their increased risk of morbidity and mortality in these patients. ${ }^{15-18}$ The present study is a subanalysis of the FARO-2 survey conducted in Italy. It is aimed at complementing the FARO project results with data regarding: the therapeutic management of SHPT in the period April 2008 to October 2008 in a subgroup of patients who started hemodialysis during the FARO project; comorbidities, hospitalizations, deaths and the percentage of patients within the ranges recommended by the Kidney Disease Outcomes Quality Initiative guidelines.
The present subanalysis evaluated the pharmaceutical costs of treatment and the evolution of bone and mineral parameters in patients new to hemodialysis who maintained the same SHPT treatment for the first 6 months following the start of dialysis.

\section{Patients and methods}

The FARO-2 was a retrospective observational survey in which all patients who had started hemodialysis treatment $\leq 8$ months earlier were enrolled. A questionnaire containing data related to clinical and laboratory parameters and to hospitalization for any reason during the week preceding each observation was filled in by the physician for each patient (all patients signed a written informed consent form as per Italian Legislative Decree 196/2003, with patient data used in an anonymous way) and the FARO-2 survey was approved by the ethical committees for each of the 26 participant centers.

The FARO-2 involved 26 of the 28 dialysis centers (two centers did not enrolled incident patients) already participating in the FARO survey; the distribution of centers was representative of the Italian national situation. A total of 568 patients were enrolled. For the purposes of this subanalysis, patients maintaining the same treatment at the beginning of dialysis and at the subsequent survey (6 months later) were selected. Patients were grouped and analyzed per type of SHTP treatment. Patients for whom no data had been collected at both observation points or who had received different SHPT treatment at the two observation points were excluded from this analysis.

Bone and mineral parameters considered for evaluation of clinical effectiveness included serum parathyroid hormone (PTH), calcium, and phosphorus. Target levels for serum PTH, calcium, and phosphorus were in accordance with the National Kidney Foundation Kidney Disease Outcomes Quality Initiative international guidelines (revised in 2003) and with the contemporary national guidelines. ${ }^{19}$ Target levels indicated by the reported guidelines were 150-300 pg/mL for intact PTH, 3.5-5.5 mg/dL for phosphorus, and $8.4-9.5 \mathrm{mg} / \mathrm{dL}$ for calcium.

Patients' demographic characteristics and data related to the presence of comorbidities, pharmacological treatments, and biochemical parameters were recorded at baseline and 6 months later (second survey). The selected subpopulations were analyzed for evolution of biochemical parameters, percentage of patients reaching target level for each biochemical parameter, SHPT drug consumption, association with other drugs, and related pharmaceutical 
costs. With regard to resource consumption, we evaluated drugs reimbursed for the treatment of SHPT, ie, phosphate binders (calcium-based and sevelamer) and erythropoietinstimulating agents (ESA).

For this cost consequence analysis, the weekly cost of pharmacological treatment per group was estimated. The analysis was performed from the Italian National Health Service perspective. Thus, for hospital drugs, the maximum approved selling prices to Italian National Health Service were considered (reference year 2008) while, for retail drugs, the approved reimbursement price was considered; all prices are pretransient compulsory reductions and include negotiated discounts published in the official Italian bulletin. Unitary costs considered was $93 €$ for an intravenous paricalcitol pack (five vials, $1 \mathrm{~mL}, 5 \mu \mathrm{g} / \mathrm{mL}$, including published negotiated discount to public structure of $7.92 \%$ ) and $5.51 €$ for an oral calcitriol pack (30 tablets, $0.25 \mu \mathrm{g}$ ); reported prices are pre every transient reduction of prices (in Italy two compulsory price reductions of 5\% and 5\% are applied to all reimbursed drugs).

Comparisons between treatment groups were assessed by measuring disease severity as baseline PTH levels; the effectiveness of treatment was expressed in terms of PTH decrease (difference from baseline to the second survey).

\section{Results}

Among the entire FARO-2 population, two prominent cohorts of patients were identified, ie, oral calcitriol $(n=105)$ and intravenous paricalcitol $(n=33)$; intravenous calcitriol $(n=5)$ and intravenous paricalcitol + cinacalcet combination $(n=2)$ cohorts were not analyzed because of the small size of the samples. In both groups analyzed, the majority of patients were males and the average age was slightly higher in the intravenous paricalcitol group versus the calcitriol group (68.7 versus 65.6 years). Baseline demographic characteristics for the different treatment groups at the start of dialysis are summarized in Table 1.

In terms of comorbidities, as reported in Table 1, hypertension and cancer were more frequent in patients on oral calcitriol, whereas cardiac pathologies, dyslipidemia, and diabetes were more frequent in patients on intravenous paricalcitol.

As reported in Table 2, the baseline biochemical profiles of patients in the two cohorts were significantly different with regard to PTH level (according to Kidney Disease Outcomes Quality Initiative guideline targets; $P<0.0001$ ), phosphorus level at baseline $(P=0.0041)$, percentage of patients at target for PTH level at baseline $(P<0.0001)$, and percentage
Table I Demographic characteristics

\begin{tabular}{lll}
\hline & PO calcitriol & IV paricalcitol \\
\hline $\begin{array}{l}\text { Patients with same SHPT } \\
\text { therapy (n) }\end{array}$ & I05 & 33 \\
$\begin{array}{l}\text { Age, years (SD) } \\
\text { Sex, } \mathrm{n}(\%)\end{array}$ & $65.5(\mathrm{I} 6.4)$ & $68.7(\mathrm{I} 6.4)$ \\
$\quad$ Females & $33(31.1 \%)$ & $1 \mathrm{I}(33.3 \%)$ \\
$\quad$ Males & $73(68.9 \%)$ & $22(66.7 \%)$ \\
Baseline comorbidities (\%) & & \\
Hypertension & 81.1 & 66.7 \\
Vascular pathology & 22.6 & 27.3 \\
Cardiac pathology & 36.8 & 48.5 \\
Liver pathology & 3.8 & 3.0 \\
Dyslipidemia & 17.9 & 39.4 \\
Cancer & 5.7 & 3.0 \\
Diabetes & 20.8 & 30.3 \\
\hline
\end{tabular}

Abbreviations: PO, oral; IV, intravenous; SD, standard deviation; SHPT, secondary hyperparathyroidism.

of patients at target for phosphorus level $(P=0.009)$. No significant differences were reported for calcium levels and percentages of patients at target for this parameter at baseline. PTH levels decreased significantly from baseline to the second survey in both groups $(P=0.0024$ in the oral calcitriol group and $P=0.0025$ in the intravenous paricalcitol group), calcium levels increased significantly in both groups ( $P=0.0046$ in the oral calcitriol group and $P=0.0255$ in the intravenous paricalcitol group), and phosphorus levels did not change significantly between the two observation points.

At the second survey, the percentage of patients reported to be at target for PTH was slightly higher in the intravenous paricalcitol group than in the oral calcitriol group, despite a significantly lower percentage of intravenous paricalcitol patients than oral calcitriol patients being at target at baseline ( $18.2 \%$ versus $34.4 \%$, respectively, $P<0.0001$ ); percentages of patients at target for phosphorus showed a trend towards an increase in the intravenous paricalcitol group, but a slight decrease in the oral calcitriol group (at the first survey, the percentage of patients at target for phosphorus was significantly higher in the oral calcitriol group $[P=0.009]$, without a significant difference at the second survey). Percentages of patients at target for calcium increased slightly in both groups, without significant differences versus baseline. Moreover, between the first and second survey, intravenous paricalcitol significantly increased the percentage of patients at target for PTH $(18.2 \%$ at baseline versus $45.5 \%$ at the second survey; $P=0.033$ ) and significantly increased the percentage of patients at target for the combined endpoint of PTH, calcium, and phosphorus ( $3.0 \%$ to $12.1 \% ; P=0.001$ ).

Weekly pharmaceutical costs for SHPT treatment per patient accounted for $1.23 €$ at baseline and $1.20 €$ at the second 
Table 2 Biochemical parameters

\begin{tabular}{|c|c|c|c|c|}
\hline & \multicolumn{2}{|c|}{ PO calcitriol $(n=105)$} & \multicolumn{2}{|c|}{ IV paricalcitol $(n=33)$} \\
\hline & $\begin{array}{l}\text { Baseline, } \\
\text { mean (SD) }\end{array}$ & $\begin{array}{l}\text { Second survey, } \\
\text { mean (SD) }\end{array}$ & $\begin{array}{l}\text { Baseline, } \\
\text { mean (SD) }\end{array}$ & $\begin{array}{l}\text { Second survey, } \\
\text { mean (SD) }\end{array}$ \\
\hline PTH level (pg/mL) & $289.4^{\mathrm{a}, \mathrm{e}}(168.4)$ & $237.2^{\mathrm{e}}(128.8)$ & $503.9^{a, f}(291.9)$ & $348.7^{f}(170.6)$ \\
\hline Ca level (mg/dL) & $8.7^{g}(0.6)$ & $8.98(0.6)$ & $8.7^{\mathrm{h}}(0.7)$ & $8.9^{\mathrm{h}}(0.6)$ \\
\hline P level (mg/dL) & $4.7^{\mathrm{b}}(\mathrm{I} . \mathrm{I})$ & $5.0(1.2)$ & $5.3^{\mathrm{b}}(\mathrm{I} .2)$ & $5.3(I . I)$ \\
\hline $\mathrm{Ca} \times \mathrm{P}$ & $40.9(10)$ & $44.4(10.9)$ & $46.3(11.9)$ & $47.3(10.9)$ \\
\hline$\%$ patients at target for PTH & $34.4 \%^{\mathrm{c}}$ & $42.6 \%$ & $18.2 \%$,i & $45.5 \%^{i}$ \\
\hline$\%$ patients at target for $\mathrm{Ca}$ & $50.0 \%$ & $56.2 \%$ & $42.4 \%$ & $45.5 \%$ \\
\hline$\%$ patients at target for $\mathrm{P}$ & $65.7 \%^{\mathrm{d}}$ & $61.0 \%$ & $39.4 \%^{\mathrm{d}}$ & $51.5 \%$ \\
\hline$\%$ patients at target $\mathrm{PTH}, \mathrm{Ca}$ and $\mathrm{P}$ & $13.2 \%$ & $13.2 \%$ & $3.0 \%$ & $12.1 \%$ \\
\hline
\end{tabular}

Notes: ${ }^{a} P<0.0001,{ }^{b} P=0.0041,{ }^{c} P<0.0001,{ }^{d} P=0.009,{ }^{e} P=0.0024,{ }^{f} P=0.0025,{ }^{g} P=0.0046,{ }^{h} P=0.0255,{ }^{i} P=0.033,{ }^{i} P<0.001$.

Abbreviations: PO, oral; IV, intravenous; SD, standard deviation; PTH, parathyroid hormone; Ca, calcium; P, phosphorus.

survey for oral calcitriol, and $42.97 €$ at baseline versus $37.46 €$ at the second survey for intravenous paricalcitol.

Considering that phosphate binders (calcium-based and sevelamer) and ESA are a substantial part of the treatment of SHPT patients, the overall costs of treatment were calculated (Table 3 ). Total weekly costs per patient, including for vitamin D, ESA, and phosphate binders, accounted for $186.32 €$ at baseline and $180.51 €$ at the second survey for oral calcitriol and for $219.94 €$ at baseline and $198.79 €$ at the second survey for intravenous paricalcitol.

The total cost of SHPT treatment was not significantly lower in the oral calcitriol group than in the intravenous paricalcitol group, either at the beginning of dialysis or 6 months later, with a difference between groups that decreased by
$46 \%$ between the two observations ( $186.32 €$ versus $219.94 €$ at baseline, $P=0.058 ; 180.51 €$ versus $198.79 €$ after 6 months, $P=0.325$, respectively). The cost of ESA at the second survey was lower $(-22 \%)$ in the intravenous paricalcitol group than in the oral calcitriol group (132.13€ versus $168.36 €$, respectively), although statistical significance was not reached $(P=0.061)$. The percentages of patients treated with ESA and average daily doses were lower in the intravenous paricalcitol group than in the oral calcitriol group both at baseline and at the second survey.

\section{Discussion}

In terms of effectiveness of the two treatments, both oral calcitriol and intravenous paricalcitol led to a significant

Table 3 Cost analysis in pateints treated with oral calcitriol or intravenous paricaclcitol

\begin{tabular}{|c|c|c|c|c|}
\hline & \multicolumn{2}{|c|}{ PO calcitriol $(n=\mid 05)$} & \multicolumn{2}{|c|}{ IV paricalcitol $(n=33)$} \\
\hline & Baseline & Survey 2 & Baseline & Survey 2 \\
\hline SHPT treatment $(€)$ & 1.23 & 1.20 & 42.97 & 37.46 \\
\hline \multicolumn{5}{|l|}{ Sevelamer } \\
\hline Percentage of patients treated & $18.9 \%$ & $19.8 \%$ & $63.6 \%$ & $69.7 \%$ \\
\hline Average (SD) daily dosage, $\mathrm{mg}$ & $2,600.00(1,066.20)$ & $3,733.33(2,151.6)$ & $2,79 \mid .40(1,2 \mid 7.0)$ & $3,272.70(1,499.90)$ \\
\hline $\begin{array}{l}\text { Average weekly expenditure for } \\
\text { percentage of patients treated }(€)\end{array}$ & 6.21 & 9.34 & 23.87 & 28.82 \\
\hline \multicolumn{5}{|l|}{ Calcium-based phosphate binders } \\
\hline Percentage of patients treated & $65.1 \%$ & $66.0 \%$ & $27.3 \%$ & $18.2 \%$ \\
\hline Average (SD) daily dosage, mg & I,94I.20 (I,024.10) & $2,097.80(1,156.2)$ & I,687.50 (593.9) & I,800.00 (758.30) \\
\hline $\begin{array}{l}\text { Average weekly expenditure for } \\
\text { percentage of patients treated }(€)\end{array}$ & 1.47 & 1.61 & 0.53 & 0.38 \\
\hline \multicolumn{5}{|l|}{ Epoietin } \\
\hline Percentage of patients treated & $96.2 \%$ & $93.4 \%$ & $87.9 \%$ & $84.8 \%$ \\
\hline Average (SD) daily dosage, IU & $10,064.00(7,696.60)$ & $9,837.00(7,632.80)$ & $9,472.40(5,339.2)$ & $8,503.20(8, \mid 44.5)$ \\
\hline $\begin{array}{l}\text { Average weekly expenditure for } \\
\text { percentage of patients treated }(€)\end{array}$ & $|77.4|$ & 168.36 & 152.57 & 132.13 \\
\hline Total weekly cost of treatment $(€)$ & 186.32 & 180.51 & 219.94 & 198.79 \\
\hline
\end{tabular}

Abbreviations: PO, oral; IV, intravenous; SD, standard deviation; SHPT, secondary hyperparathyroidism. 
reduction in PTH levels, but only intravenous paricalcitol led to a significant increase in the percentage of patients at target for PTH levels (from 18.2\% at baseline to $45.5 \%$ at second survey; $P=0.033$ ) and to a significant increase in the percentage of patients at target at the same time points for $\mathrm{PTH}$, calcium, and phosphorus (from 3.0\% at baseline to $12.1 \%$ at second survey; $P=0.001$ ).

This study highlights that costs related to health care resource utilization, as already reported in a European Union study ${ }^{20}$ and a US study, ${ }^{21}$ increased with rising baseline PTH. Confirming the findings of the cost consequences analysis of the FARO survey ${ }^{22}$ performed on the overall hemodialysis population, the present study showed that different baseline severity of SHPT in terms of PTH level was associated with different treatment patterns. Oral calcitriol, in fact a low cost therapeutic option, was used effectively in significantly less severe patients than intravenous paricalcitol (see Table 2) in terms of baseline PTH level and of baseline percentages of patients at target for all bone mineral parameters (PTH, calcium, and phosphorus) that were higher in the oral calcitriol group than in the intravenous paricalcitol group. Despite these differences in disease severity between the two groups at baseline (PTH level at baseline was almost double in the intravenous paricalcitol group compared with the oral calcitriol group), the total pharmaceutical weekly costs (including for vitamin D, ESA, and phosphate binders) were similar in the two groups, with a difference that decreased between baseline and the second survey.

This study also highlights the relevance in terms of costs of therapies associated with vitamin D in the treatment of SHPT (ESA and phosphate binders), ie, oral calcitriol therapy accounted for about $0.7 \%$ of total costs, while intravenous paricalcitol accounted for about $19 \%$ of total costs both at baseline and at the second survey.

The structure of the FARO-2 study is the most important limitation of the present analysis. The study was not controlled and lacks comparability between cohorts, although it reflects the treatment pathways at the time of the survey. Moreover, a priori, we hypothesized that patients who remained on the same treatment between baseline and the second survey would remain on the same treatment in the period between the two observations (in fact, because data were collected in one survey every 6 months, there was no indication of resource consumption for the period between the two surveys). Finally, the selection of cohorts of patients who remained on the same treatment between the two surveys resulted in a reduction of sample size, but allows an economic evaluation for the different treatment cohorts. It should also be considered that, because these patients are frequently on multidrug therapy, their compliance with oral drugs could be suboptimal and could negatively affect the efficacy of oral pharmacological therapies.

Other important limitations of this study are related to the survey design, in that it was not controlled and lacked comparability between cohorts; however, it is reflective of real Italian practice patterns at the time of the study. Finally, considering the relatively small sample size, further studies of this topic in a wider population could be helpful to confirm the results of the present analysis.

\section{Conclusion}

The present analysis, performed in a subpopulation of the FARO-2 survey, was conducted to evaluate the biochemical consequences and overall costs of pharmacological treatment in patients new to dialysis and affected by SHPT. Among the two identified groups, only intravenous paricalcitol significantly increased the percentage of patients at target for the combined endpoint PTH, Ca, and P $(p=0.001)$ and the total cost of treatment of the intravenous paricalcitol patients after six months from dialysis entrance was not significantly higher than in the oral calcitriol patients.

\section{Author contributions}

All the authors conceived and performed the present analysis and have written the manuscript.

\section{Disclosure}

An abstract reporting the preliminary results of this study was presented as a poster at the 49th European Renal AssociationEuropean Dialysis and Transplant Association Congress, May 24-27, 2012, Paris, France. The design, study conduct, and financial support for the clinical trial was provided by AbbVie. AbbVie participated in interpretation of the data, review, and approval of the manuscript.

Daniela Paola Roggeri is a consultant for AbbVie. Piergiorgio Messa is a member of the FARO Steering Committee, and has received lecture honoraria from Janssen Cilag, and Amgen. Mario Cozzolino is a member of the FARO Steering Committee, and has received lecture honoraria from AbbVie, Shire, Amgen, Sanofi/Genzyme, Vifor-Fresenius, and Roche. Diego Brancaccio is a member of the FARO Steering Committee, is a consultant for Abbott, and has received lecture honoraria from GSK, Amgen, and Shire. Ernesto Paoletti is a consultant for and has received 
honoraria from AbbVie, Novartis, and Janssen-Cilag. Alessandro Roggeri is a consultant for AbbVie. Anna Maria Costanzo is a Head of Medical Affairs SH, for AbbVie Italy. Umberto di Luzio Paparatti is an Affiliate Medical Director for AbbVie, Italy. Vincenzo Festa is a Medical Manager for AbbVie Italy. Sandro Mazzaferro is a member of the FARO Steering Committee, and has received lecture honoraria from Shire and Amgen.

\section{References}

1. Centers for Disease Control and Prevention. Prevalence of chronic kidney disease and associated risk factors - United States. Available from: http://www.cdc.gov/mmwr/preview/mmwrhtml/m m5608a2.htm. Accessed July 24, 2014.

2. Italian Registry of Dialysis and Transplantation. Report 2010. Available from: http://www.sin-ridt.org/web/eventi/RIDT/registro_italiano.cfm. Accessed July 24, 2014.

3. Jansen DL, Heijmans MJ, Rijken M, et al. Illness perceptions and treatment perceptions of patients with chronic kidney disease: different phases, different perceptions? Br J Health Psychol. 2013;18(2):244-262.

4. Wyld M, Morton RL, Hayen A, Howard K, Webster AC. A systematic review and meta-analysis of utility-based quality of life in chronic kidney disease treatments. PLoS Med. 2012;9(9):e1001307.

5. Mujais SK, Story K, Brouillette J, et al. Health-related quality of life in CKD patients: correlates and evolution over time. Clin J Am Soc Nephrol. 2009;4(8):1293-1301.

6. Abdel-Kader K, Myaskoversusky L, Karpov I, et al. Individual quality of life in chronic kidney disease: influence of age and dialysis modality. Clin J Am Soc Nephrol. 2009;4(4):711-718.

7. Procaccini DA, Angelini P, Aucella F, et al; on behalf of Apulia Nephrology Quality of Life Study. [Health-related quality of life in patients with chronic kidney disease]. G Ital Nefrol. 2008;25(6):694-671. Italian.

8. Chronic Kidney Disease Initiative. Protecting kidney health. Available from: http://www.cdc.gov/diabetes/projects/pdfs/CKD_Factsheet.pdf. Accessed July 24, 2014.

9. Levey AS, Atkins R, Coresh J, et al. Chronic kidney disease as a global public health problem: approaches and initiatives - a position statement from Kidney Disease Improving Global Outcomes. Kidney Int. 2007;72(3):247-259.
10. Joy MS, Karagiannis PC, Peyerl FW. Outcomes of secondary hyperparathyroidism in chronic kidney disease and the direct costs of treatment. J Manag Care Pharm. 2007;13(5):397-411.

11. Fondazione CENSIS. [Substitution treatment of renal function in Italy: clinical, economic and social aspects]. Available from: http:// www.censis.it/14?shadow_ricerca=5688. Accessed July 24, 2014. Italian.

12. Cicchetti A, Ruggeri M, Codella P, Ridolfi A. [The social and health costs of chronic renal failure]. Farmeconomia e Percorsi Terapeutici. 2011;12(1):21-28. Italian.

13. Jeantet A, Piccoli GB, Pacitti A, et al. [Costs of dialysis in hospitalised patients with acute or chronic renal failure, according to area of treatment]. G Ital Nefrol. 2002;19(3):308-315. Italian.

14. Pontoriero G, Pozzoni P, Vecchio LD, Locatelli F. International study of health care organization and financing of renal replacement therapy in Italy: an evolving reality. Int $J$ Health Care Finance Econ. 2007;7(2-3):201-215.

15. Tomasello S. Secondary hyperparathyroidism and chronic kidney disease. Diabetes Spectr. 2008;21(1):19-25.

16. Block GA, Klassen PS, Lazarus JM, Ofsthun N, Lowrie EG, Chertow GM. Mineral metabolism, mortality, and morbidity in maintenance hemodialysis. J Am Soc Nephrol. 2004;15(8):2208-2218.

17. Floege J, Kim J, Ireland E, et al. Serum iPTH, calcium and phosphate, and the risk of mortality in a European haemodialysis population. Nephrol Dial Transplant. 2011;26(6):1948-1955.

18. Coen G, Manni M, Mantella D, et al. Are PTH serum levels predictive of coronary calcifications in haemodialysis patients? Nephrol Dial Transplant. 2007;22(11):3262-3267.

19. Mazzaferro S, Cozzolino M, Marangella M, Strippoli GF, Messa P. [Calcimimetics, phosphate binders, vitamin $\mathrm{D}$ and its analogues for treating secondary hyperparathyroidism in chronic kidney disease: guideline from the Italian Society of Nephrology]. G Ital Nefrol. 2007;24 Suppl 37:107-124. Italian.

20. Chiroli S, Mattin C, Belozeroff V, Perrault L, Mitchell D, Gioni I. Impact of mineral and bone disorder on healthcare resource use and associated costs in the European Fresenius medical care dialysis population: a retrospective cohort study. BMC Nephrol. 2012;13:140.

21. Schumock GT, Andress DL, Marx SE, Sterz R, Joyce AT, KalantarZadeh K. Association of secondary hyperparathyroidism with CKD progression, health care costs and survival in diabetic predialysis CKD patients. Nephron Clin Pract. 2009;113(1):c54-c61.

22. Roggeri DP, Mazzaferro S, Brancaccio D, et al. Pharmacological control of secondary hyperparathyroidism in hemodialysis subjects: a cost consequences analysis of data from the FARO study. $J$ Med Econ. 2012;15(6):1-8.

\section{Publish your work in this journal}

The International Journal of Nephrology and Renovascular Disease is an international, peer-reviewed open-access journal focusing on the pathophysiology of the kidney and vascular supply. Epidemiology, screening, diagnosis, and treatment interventions are covered as well as basic science, biochemical and immunological studies. The journal welcomes original research, clinical studies, reviews \& evaluations, expert opinion and commentary, case reports and extended reports. The manuscript management system is completely online and includes a very quick and fair peerreview system, which is all easy to use. Visit http://www.dovepress.com/ testimonials.php to read real quotes from published authors. 